\title{
SOFT X-RAY TRANSIENTS ARE LIKE ER UMA CATACLYSMIC VARIABLES
}

\author{
ENIKŐ REGÖS \\ Department of Mathematics, Monash University, Clayton, Victoria 3168, Australia. \\ AND \\ NICOLA MASETTI \\ Dipartimento di Astronomia, Universit di Padova, vicolo dell'Osservatorio, 5 I-35122 \\ Padua, Italy
}

\begin{abstract}
We discuss how soft X-ray transients exhibit light curves associated with two accretiondisc instabilities that are well known to operate in cataclysmic variables. A thermal instability that is due to hydrogen ionization leads to the transient nature but is modified by $\mathrm{X}$-ray irradiation of the disc. Growth of the disc between the ensuing outbursts leads to a $3: 1$ commensurability instability at the outer edge of the disc that drives superoutbursts and produces superhumps in the lightcurve if the mass ratio is sufficiently small. Superhumps are observed only in the short period SU UMa CVs but black hole primaries allow SXTs to exhibit superoutbursts and superhumps at much larger periods. We point out similarities between SXT superhump shapes and those of the small class of ER UMa CVs. The similarity between these systems stems from the fact that the mass-transfer rates are almost large enough to stabilize the disc against the thermal instability in both cases.
\end{abstract}

\section{Introduction}

We illustrate how CV type depends on mass-transfer rate and orbital period in fig 1. Cataclysmic Variables have a white dwarf primary and normally a low-mass main-sequence secondary that is filling its Roche lobe. Dwarf Novae (U Geminorum) stars have discs which are thermally unstable and show outbursts. Fig 2 shows how the disc structure swaps between high and low states when the average rate of mass transfer lies on an unstable branch (shown dashed) of the S-curve, the shape of which is due to hydrogen ionization. High mass transfer rates stabilize the discs - stable nova-like systems above the solid curved line. Z Cam systems are borderline. For mass ratios less than 0.25 the disc can grow out to the $3: 1$ commensurability resonance and become unstable to orbit crossing (Whitehurst \& King 1991). For a $1 M_{\odot}$ white dwarf, because of the relation between secondary mass and period, SU Ursae Majoris systems to the left of the solid vertical line are unstable in this way and show superoutbursts (fig 3) with superhumps visible in the light curve at maximum. The dotted vertical lines indicate the location of the period gap in the CV distribution. ER UMa systems are at the border between SU UMa systems and those with thermally stable discs which still show superhumps.

$\mathrm{X}$-ray binaries (fig 4) are like cataclysmic variables but have a neutron star or black hole in place of the white dwarf primary. The dashed curved line is the equivalent of the yellow thermal stability line for CVs. The accretion discs in X-ray binaries are irradiated by X-radiation from the central object which changes the disc structure. The stability line is lowered to the solid curved line (van Paradijs 1996). The dashed vertical line is the period at which a $1.8 M_{\odot}$ neutron star would have a main-sequence secondary 0.25 times its mass. The solid vertical line is the same for a $7 M_{\odot}$ black hole. It is easier to get a mass ratio small enough for superhumps. Transient systems lie below the yellow line while persistent sources lie above it. 


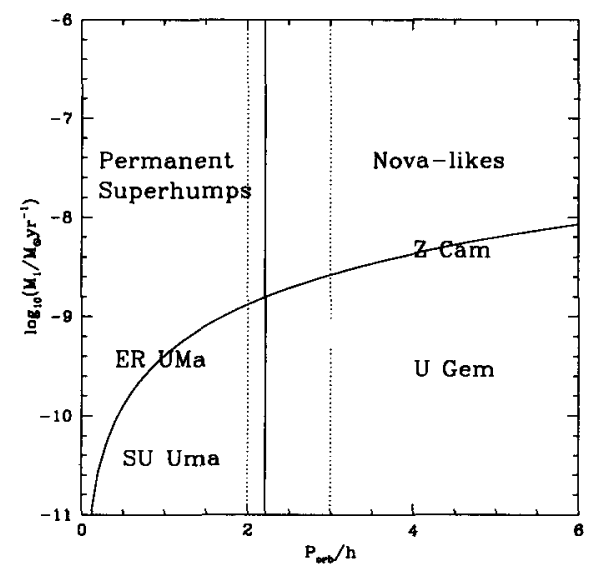

Figure 1. Mass-transfer rate - orbital period diagram for $\mathrm{CVs}$

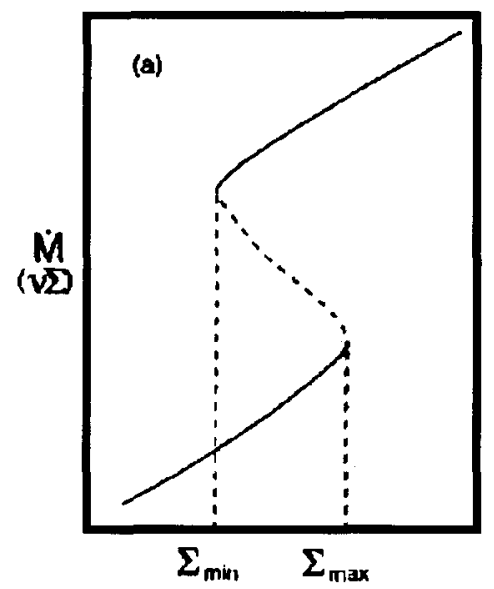

Figure 2. Mass-transfer rate (proportional to the product of the viscosity $\nu$ and surface density $\Sigma$ ) against surface density in a disc annulus - the S-curve (Osaki 1996)

\section{Superhump lightcurves}

Superhumps with a period slightly larger than the orbital period appear superimposed on the superoutburst lightcurves of SU UMa systems. Fig 5 shows the steep rise and gentle decline found for all normal SU UMa stars and for ER UMa stars at the start of a superoutburst. Fig 6 shows the opposite shape normally found for ER UMa stars towards the end of the superoutburst. Fig 7, to be compared with fig 6 shows the similar shape found for SXTs.

\section{Conclusions}

Comparing the properties and the superhump lightcurves of SXTs with ER Uma systems we deduce that they are borderline between persistent and transient sources. This is consistent with work of King, Kolb and Burderi (1996) which indicates that SXTs have difficulty lying below the stability line. They must be slightly evolved to do so. They are therefore more likely to lie on it than well 

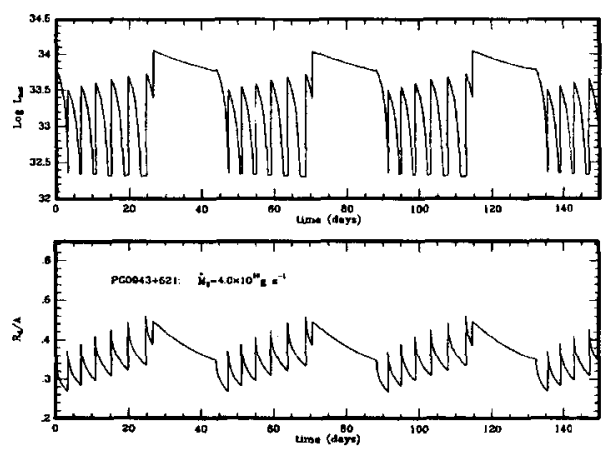

Figure 3. Outburst cycles in a typical SU UMa system. The upper panel shows the luminosity variation while the lower panel shows how the radius grows to a maximum just before a superoutburst (Osaki 1996)

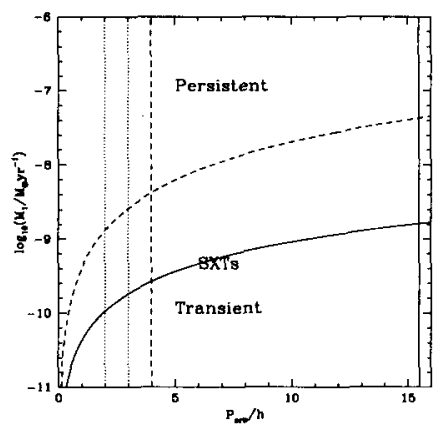

Figure 4. Mass-transfer rate - orbital period diagram for X-ray binaries

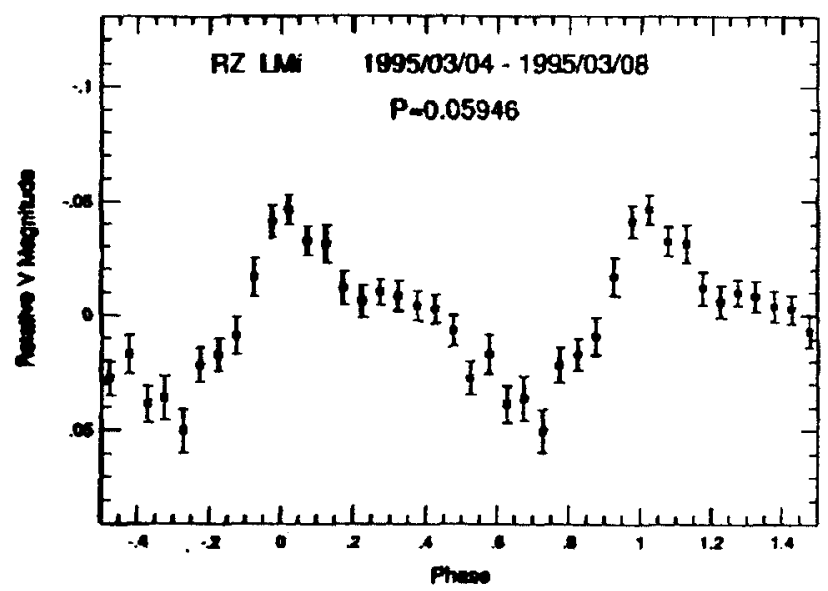

Figure 5. Superhump lightcurve of RZ LMi - ER UMa at the start of a superoutburst or normal SU UMa type (Nogami et al. 1995) 


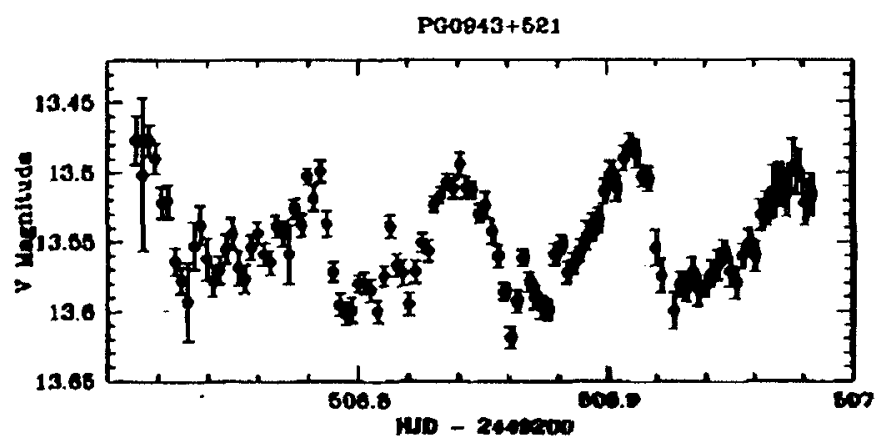

Figure 6. Superhump lightcurve of PG 0943+521 - ER UMa system towards the end of a superoutburst (Robertson et al. 1995)

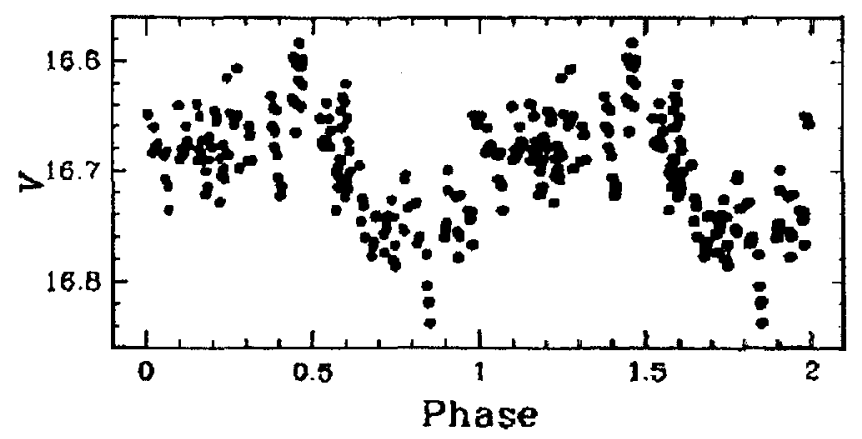

Figure 7. Superhump lightcurve of V2293 Oph - SXT (Masetti et al. 1996)

below it. Because it is difficult to get transient behaviour in SXTs, owing to the necessarily lower mass-transfer rates, we expect them to lie on, rather than below, the borderline and therefore to be similar to ER UMa stars (Masetti and Regös 1997). And indeed, this is justified in the observed superhump lightcurves.

\section{References}

King A. R., Kolb U., Burderi L., 1996, ApJ, 464, L127

Masetti N., Bianchini A., Bonibaker J., Della Valle M., Vio R., 1996, A\&A, 314, 123

Masetti N., Regös E., 1997, New Astr., in press

Nogami D., Kato T., Masuda S., Hirata R., Matsumoto K., Tanabe K., Yokoo T., 1995, PAS.J, 47, 897

Osaki Y., 1996, PASP, 108, 39

Robertson J. W., Honeycutt R. K., Turner G. W., 1995, PASP, 107, 443

van Paradijs J., 1996, ApJ, 464, L139

Whitehurst R., King A. R., 1991, MNRAS, 249,25 